\title{
Extraction of high-molecular-weight genomic DNA for long-read sequencing of single molecules
}

Baptiste Mayjonade ${ }^{1}$, Jérôme Gouzy ${ }^{1}$, Cécile Donnadieu², Nicolas Pouilly ${ }^{1}$, William Marande ${ }^{3}$, Caroline Callot ${ }^{4}$, Nicolas Langlade ${ }^{1}$, and Stéphane Muños ${ }^{1}$

'LIPM, Université de Toulouse, INRA, CNRS, Castanet-Tolosan, France, ${ }^{2}$ Get-PLAGE, Université de Toulouse, INRA, CNRS, Castanet Tolosan, France, ${ }^{3} \mathrm{CNRGV}$, Université de Toulouse, INRA, CNRS, Castanet Tolosan, France, and ${ }^{4} C R C T$, INSERM, Université de Toulouse, CNRS, Toulouse, France

BioTechniques 61:203-205 (October 2016) doi 10.2144/000114460

Keywords: DNA extraction; long-read sequencing; PacBio

Supplementary material for this article is available at www.BioTechniques.com/article/114460.

De novo sequencing of complex genomes is one of the main challenges for researchers seeking high-quality reference sequences. Many de novo assemblies are based on short reads, producing fragmented genome sequences. Third-generation sequencing, with read lengths $>10 \mathrm{~kb}$, will improve the assembly of complex genomes, but these techniques require high-molecular-weight genomic DNA (gDNA), and gDNA extraction protocols used for obtaining smaller fragments for short-read sequencing are not suitable for this purpose. Methods of preparing gDNA for bacterial artificial chromosome (BAC) libraries could be adapted, but these approaches are time-consuming, and commercial kits for these methods are expensive. Here, we present a protocol for rapid, inexpensive extraction of high-molecular-weight gDNA from bacteria, plants, and animals. Our technique was validated using sunflower leaf samples, producing a mean read length of $12.6 \mathrm{~kb}$ and a maximum read length of $80 \mathrm{~kb}$.

Next-generation sequencing (NGS) is commonly used in genomics and, until recently, only produced sequences hundreds of nucleotides long. The genomes of higher eukaryotes, especially plants, contain a large number of repeated sequences that can reach several tens of kilobases in length. De novo assemblies of large complex genomes based on short reads result in fragmented genome sequences containing thousands to millions of contigs or scaffolds (1). Thirdgeneration sequencers based on the sequencing of a single DNA molecule produce sequences of several tens of kilobases, which can improve genome assemblies because the longer reads can often span repeated regions.
High-quality and high-molecularweight DNA $(>50 \mathrm{~kb})$ is needed to fully benefit from the potential of thirdgeneration sequencers. The polymerase used for sequencing (DNA/Polymerase Binding Kit P6 v2; Pacific Biosciences, Menlo Park, CA) is highly sensitive to contaminants bound to DNA, which could result in shorter sequences.

While preparation of megabasesized DNA in an agarose gel matrix is possible (2), the process takes 3 days, and it is difficult to extract the DNA from the agarose plug. Commercial kits for the extraction of high-molecular-weight DNA are also available, but they are expensive.

Here we describe a protocol (see Supplementary Material) to extract micrograms of high-molecular-weight genomic DNA (gDNA) up to $150 \mathrm{~kb}$ length in a single microtube in under $60 \mathrm{~min}$ at a cost of $<\$ 0.20$ per sample (Figure 1). We fine-tuned this protocol for sunflower leaf samples and subsequently validated it for bacteria and human cells. Sunflowers are known to contain polyphenols and other contaminants, such as polysaccharides and tannins (3); therefore, it is challenging to extract very pure DNA from this plant species.

We harvested leaves from 3-week-old plantlets. The samples were immediately frozen in liquid nitrogen and stored at $-80^{\circ} \mathrm{C}$ until grinding to prevent DNA degradation.

The first step in the gDNA extraction consisted of mechanically and chemically disrupting cell membranes and (for plants) cells walls to release nuclei and DNA molecules. We ground the leaves into a fine powder in liquid nitrogen. For the lysis step, we used an SDS-based buffer to dissolve the cell membranes $(4,5)$. The detergent SDS neutralizes and inactivates proteins, especially endonucleases, that degrade DNA. During this step, we also included RNase A in the lysis buffer to digest RNA. During the lysis step, it is crucial to immediately homogenize the frozen ground tissue in the lysis buffer to quickly inactivate nucleases. The degree of homogenization of the lysate is also correlated with the yield

\section{METHOD SUMMARY}

We present a protocol for the rapid ( 1 hr), inexpensive, and efficient isolation of high-molecular-weight genomic DNA (gDNA) that can be used to prepare libraries for long-read sequencing on the PacBio RSII instrument. 
Table 1. Quality and quantity of genomic DNA (gDNA) samples.

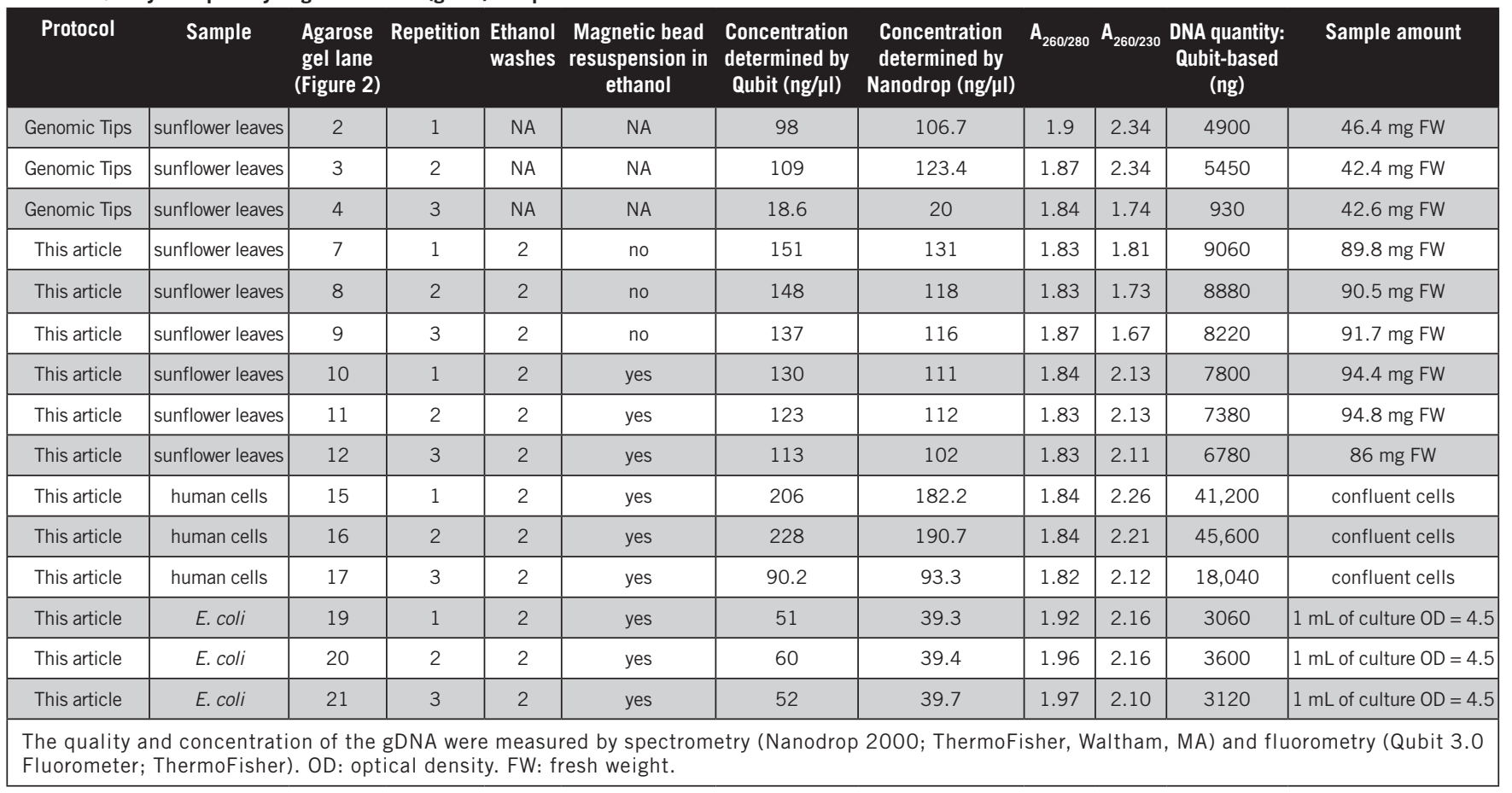

of the extraction process and the purity of the DNA. In some cases, heating the lysis buffer can help to homogenize the solution. However, heating the sample above $65^{\circ} \mathrm{C}$ for more than $1 \mathrm{~h}$ can lead to DNA damage.

At the end of the lysis step, the addition of potassium acetate to the lysis solution leads to the formation of potassium dodecyl sulfate, which is insoluble. Proteins and polysaccharides then form a complex with the potassium dodecyl sulfate precipitate, which is then removed by centrifugation (6).

The second step is purification of DNA from the lysis solution. Carboxylated magnetic beads were used because they are cost-effective (7) and cause minimal DNA shearing. Polyethylene glycol 8000 (PEG8000) was used to bind DNA to the beads, as previously described $(7,8)$. PEG8000 is safe and precipitates fewer contaminants compared with isopropanol or ethanol (9).

For the final step, gDNA was washed in $70 \%$ ethanol to remove contaminants that were poorly bound to the magnetic beads as well as the remaining reagents used in the previous steps. DNA was eluted from the beads by resuspension in a buffered solution. We used a spectrophotometric method to evaluate the purity of our DNA solutions. The $A_{260 / 230}$ ratio is affected by the presence
Table 2. Characteristics of PacBio sequences from sunflower leaf libraries with different size cutoffs.

\begin{tabular}{|c|c|c|c|c|c|c|c|}
\hline $\begin{array}{c}\text { g-TUBE fragmentation } \\
\text { parameters on Eppendorf } \\
\text { centrifuge \#5424 }\end{array}$ & $\begin{array}{c}\text { BluePippin size- } \\
\text { selection cutoff } \\
\text { (kb) }\end{array}$ & $\begin{array}{c}\text { Movie } \\
\text { length } \\
\text { (min) }\end{array}$ & $\begin{array}{c}\text { Total bases } \\
\text { (Mb/SMRT cell) }\end{array}$ & $\begin{array}{c}\text { Polymerase } \\
\text { read length } \\
\text { (bp) }\end{array}$ & $\begin{array}{c}\text { N50 } \\
\text { subread } \\
\text { (bp) }\end{array}$ & $\begin{array}{c}\text { Max } \\
\text { subread } \\
\text { (bp) }\end{array}$ \\
\hline $6000 \mathrm{~g} / 1 \mathrm{~min}$ & 12 & 240 & 1106.01 & 13,940 & 12,866 & 50,298 \\
\hline $5000 \mathrm{~g} / 1 \mathrm{~min}$ & 15 & 240 & 1109.57 & 14,740 & 15,100 & 49,331 \\
\hline $4000 \mathrm{~g} / 2 \mathrm{~min}$ & 18 & 360 & 1057.60 & 15,836 & 19,206 & 80,974 \\
\hline $3800 \mathrm{~g} / 3 \mathrm{~min}$ & 20 & 360 & 1026.39 & 17,126 & 20,507 & 73,379 \\
\hline
\end{tabular}

N50 is the value for which $50 \%$ of all bases come from subreads longer than this value. g-TUBEs were purchased from Covaris (Woburn, MA). The BluePippin instrument was purchased from Sage Science (Beverly, MA).

\section{Biological material (bacteria, cells, tissue powder)}

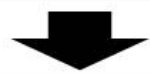

Cell lysis and RNA removal

30 minutes
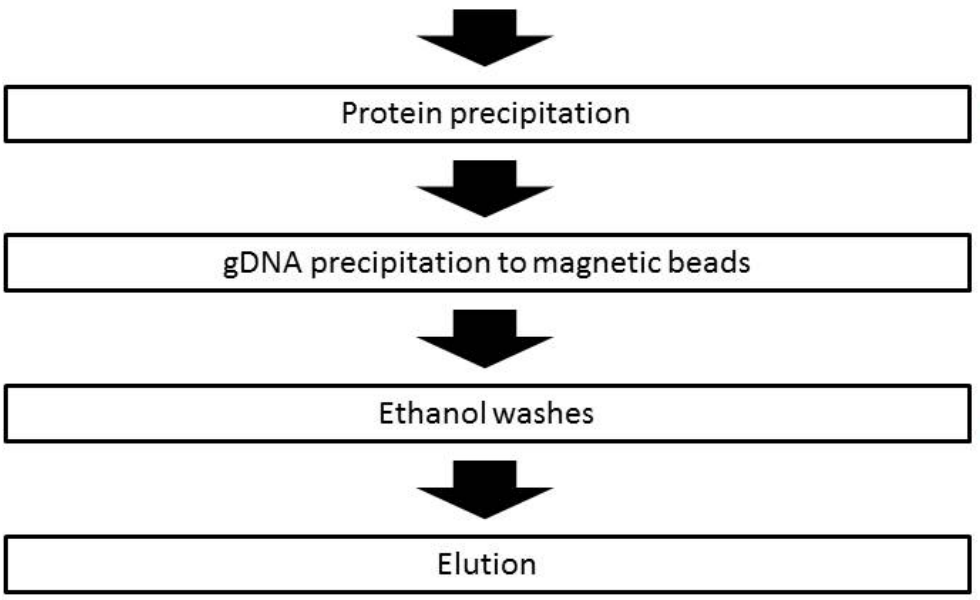

10 minutes

10 minutes

Figure 1. Genomic DNA (gDNA) extraction workflow. 

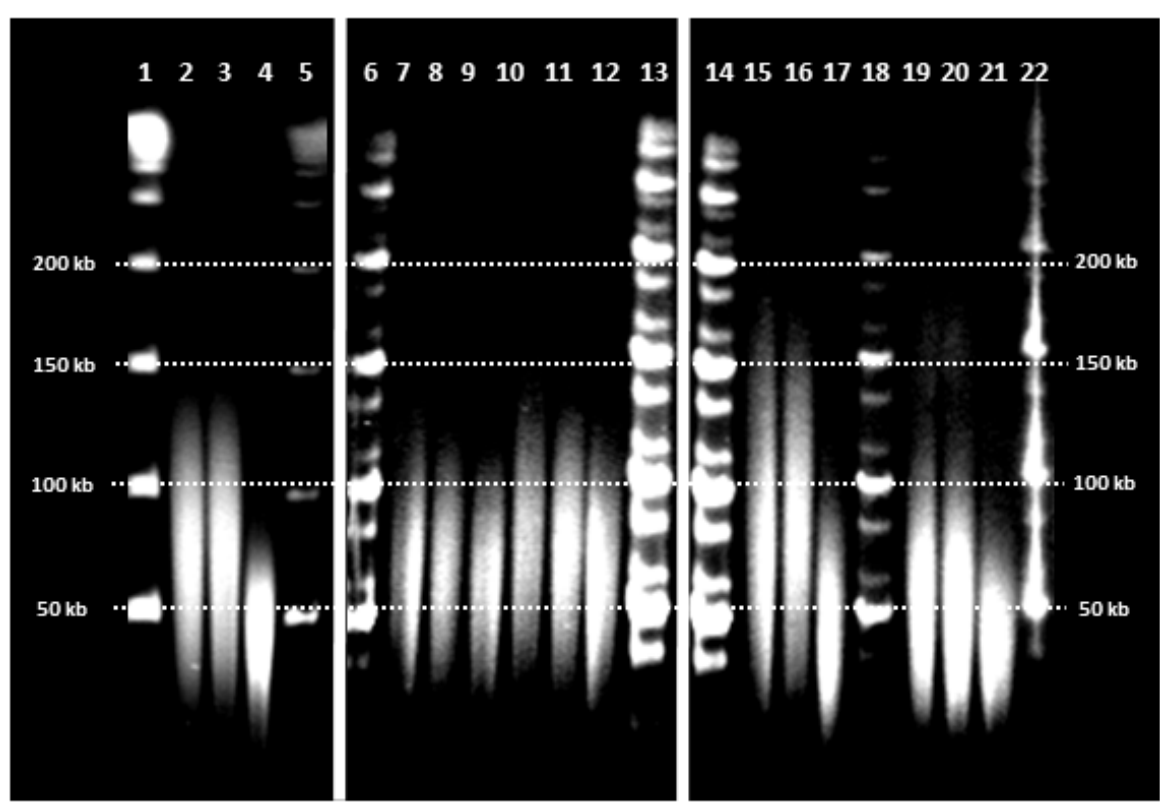

Figure 2. Pulsed field agarose gel electrophoresis of genomic DNA (gDNA). The integrity of gDNA, extracted by different protocols was evaluated on field electrophoresis agarose gels. Lanes 1 and 5: lambda PFG ladder; Lanes 6,13,14,18, and 22: MidRange I PFG marker; Lanes 2-4: sunflower gDNA extracted with QIAGEN Genomic Tips; Lanes 7-9: sunflower gDNA extracted with our protocol (two ethanol washes without bead resuspension); Lanes 10-12: sunflower gDNA extracted with our protocol (two ethanol washes with bead resuspension); Lanes 15-17: HEK293 human cell gDNA extracted with our protocol; Lanes 19-21: NEB 5-alpha electrocompetent E. Coli gDNA extracted with our protocol.

of certain contaminants. We found that the resuspension of the beads in $70 \%$ ethanol was crucial for complete removal of contaminants that absorb light at $230 \mathrm{~nm}$ (Table 1). Two additional washes could be performed without decreasing the integrity of the DNA (data not shown). We obtained an $\mathrm{A}_{260 / 280}$ ratio $>1.8$, indicating that our DNA solution was protein-free. The concentrations quantified by either spectrophotometry or fluorescent intercalating dye-based methods (Table 1) were similar, indicating that the DNA solution was pure. Our extractions yielded $6.7-7.8 \mu \mathrm{g}$ of highly pure DNA from $90 \mathrm{mg}$ of fresh sunflower leaves.

We evaluated the integrity of the extracted gDNA by performing pulsedfield electrophoresis, which showed that the DNA molecules ranged in size from $\sim 20 \mathrm{~kb}$ to $\sim 130 \mathrm{~kb}$ (Figure 2).

Comparing DNA solutions extracted using our protocol to DNA extracted with the Genomic Tips 20/G commercial kit (QIAGEN, Hilden, Germany), it can be seen that the purity and integrity of the gDNA were similar for both protocols (Table 1 and Figure 2). However, the commercial kit is 35 times more expensive (\$7 per sample) and requires at least $3 \mathrm{~h}$ to complete the extraction, compared with $1 \mathrm{~h}$ for our protocol.
We next evaluated the suitability of our protocol for gDNA extraction from bacteria and animal cells (E. coli and human cell culture, respectively). Highmolecular-weight gDNA, with similar characteristics to the sunflower gDNA we obtained, was successfully extracted (Table 1 and Figure 2).

Finally, we validated the quality of sunflower gDNA extracted with our protocol by sequencing with the PacBio RS II (Pacific Biosciences, Menlo Park, California) using 4 different PacBio libraries with 4 DNA size cutoffs ranging $12-20 \mathrm{~kb}$ (Table 2). No inhibition of the sequencing reaction was observed because the sequence reads were consistent with PacBio specifications (10-15 kb for 240-min-long movies and up to 17,126 bp for 360-min-long movies). These sequencing results confirmed that the gDNA was pure and of high molecular weight. According to Pacific Biosciences specifications and the activity of the P6 polymerase, the maximum read length that can be obtained is limited to 46,000 bp with a 240-min-long movie. With a 240-min-long movie, we obtained sub-reads (i.e., inserts of gDNA) of up to $50,298 \mathrm{bp}$, confirming the quality of the gDNA extracted with our protocol. The maximal length of the sub-reads increased to 80,974 bp with a movie length of $360 \mathrm{~min}$.

\section{Author contributions}

B.M. contributed to the development of the protocol, the experimental procedures and the writing of the manuscript. J.G. contributed to the bioinformatic analysis of the sequencing data and the writing of the manuscript. N.P., C.D., W.M. and C.C. participated in the experimental procedures. N.L., coordinator of the SUNRISE project, participated in the writing of the manuscript. S.M. participated in the coordination of the work and the writing of the manuscript.

\section{Acknowledgments}

This research was funded by the SUNRISE project (wWW.sunrise-project.fr; Project ANR-11-BTBR-0005).

\section{Competing interests}

The authors declare no competing interests.

\section{References}

1. Treangen, T.J. and S.L. Salzberg. 2011. Repetitive DNA and next-generation sequencing: computational challenges and solutions. Nat. Rev. Genet. 13:36-46.

2. Zhang, M., Y. Zhang, C.F. Scheuring, C.C. Wu, J.J. Dong, and H.B. Zhang. 2012. Preparation of megabase-sized DNA from a variety of organisms using the nuclei method for advanced genomics research. Nat. Protoc. 7:467-478.

3. Horne, E.C., S.P. Kumpatla, K.A. Patterson, M. Gupta, and S.A. Thompson. 2004. Improved highthroughput sunflower and cotton genomic DNA extraction and PCR fidelity. Plant Mol Biol Report. 22:83-84.

4. Dellaporta, S.L., J. Wood, and J.B. Hicks. 1983. A plant DNA minipreparation: version II. Plant Mol Biol Report. 1:19-21.

5. Tai, T. H. and S.D. Tanksley. 1990. A rapid and inexpensive method for isolation of total DNA from dehydrated plant tissue. Plant Mol Biol Report. 8:297-303

6. Bryant, J.L., P.M. Dey, and J.B. Harborne. 1996. Methods in Plant Biochemistry, Volume 10B: Molecular Biology. Molecular Biology. Academic Press, Cambridge, MA.

7. Rohland, N. and D. Reich. 2012. Cost-effective, high-throughput DNA sequencing libraries for multiplexed target capture. Genome Res. 22:939-946.

8. Hawkins, T.L., T. O'Connor-Morin, A. Roy, and C. Santillan. 1994. DNA purification and isolation using a solid-phase. Nucleic Acids Res. 22:4543-4544.

9. Shan, G., W. Jin, E.K. Lam, and X. Xing. 2008 Purification of total DNA extracted from activated sludge. J. Environ. Sci. (China) 20:80-87.

Received 24 May 2016; accepted 22 July 2016.

Address correspondence Baptiste Mayjonade, LIPM Université de Toulouse, INRA, CNRS, Castanet-Tolosan, France. E-mail: Baptiste.Mayjonade@toulouse.inra.fr

To purchase reprints of this article, contact: biotechniques@fosterprinting.com 